\title{
Dynamics of fertility in patients on waiting list for kidney transplantation
}

\author{
Zachoval $\mathrm{R}^{1}$, Jarabak $\mathrm{J}^{1}$, Slatinska $\mathrm{J}^{2}$, Burgelova $\mathrm{M}^{2}$, Sobotka $\mathrm{V}^{3}$, Vranova $\mathrm{J}^{4}$, Krhut $\mathrm{J}^{5}$ \\ Department of Urology, Thomayer Hospital and First Faculty of Medicine, Charles University, Prague, \\ Czech Republic. roman.zachoval@ftn.cz
}

\begin{abstract}
Objectives: To evaluate the presence of hormonal abnormalities and fertility disorders in patients with chronic kidney disease (CKD) awaiting renal transplantation.

Methods: From September 2009 to April 2011 all male patients with CKD awaiting kidney transplantation were investigated. The following tests were performed: semen analysis, serum concentration of testosterone, SHBG, $\mathrm{LH}, \mathrm{FSH}$ and prolactin. Differences in hormone levels and sperm count parameters were statistically evaluated between the control group and the patient group.

Results: The group of patients consisted of 74 and the control group of 41 men. Average testosterone levels were lower in patients compared to control group. In patients significantly higher levels of SHBG, LH, FSH and PRL were found, and statistically significantly lower ejaculate volume, total sperm count, sperm concentration, total and progressive sperm motility and sperm morphology than in the control group. Within the group of patients a negative correlation between testosterone and PRL was found and a positive correlation between testosterone and total sperm motility and morphology. A negative correlation was detected between the duration of haemodialysis and testosterone, sperm concentration, total and progressive motility and sperm morphology. Conclusion: Significant changes in hormone levels and impaired fertility are found in haemodialyzed patients on a waiting list for kidney transplantation. The dynamics of these changes are dependent on the duration of haemodialysis (Tab. 4, Fig. 2, Ref. 15). Text in PDF www.elis.sk.

Key words: chronic kidney disease, fertility, haemodialysis, kidney transplantation, spermiogram.
\end{abstract}

In the Czech Republic 6318 patients were dependent on dialysis at the end of 2010, i.e. 602 per 1 million inhabitants. Haemodialysis was performed in 5820 patients and peritoneal dialysis in 498 patients. Number of haemodialysis patients increases every year, 379 haemodialysis patients/1 million inhabitants were registered in 2001, and in 2010 it was already 554 patients/1 million inhabitants. Of the total number of haemodialysis patients, $30 \%$ is under the age of 60 and approximately $20 \%$ of haemodialysis patients should be considered for renal transplantation according to current standards (1).

Approximately $15 \%$ of couples are unable to conceive within 1 year and seek treatment for infertility. Semen analysis abnormalities (oligozoospermia, asthenozoospermia and teratozoospermia) contribute or cause one half of these cases. Male fertility may

\footnotetext{
'Department of Urology, Thomayer Hospital and First Faculty of Medicine, Charles University, Prague, Czech Republic, ${ }^{2}$ Nephrology Department, Institute for Clinical and Experimental Medicine, Prague, Czech Republic, ${ }^{3}$ Sanatorium Pronatal, Prague, Czech Republic, ${ }^{4}$ Department of Medical Biophysics and Informatics, Third Faculty of Medicine, Charles University, Prague, Czech Republic, and ${ }^{5}$ Department of Urology, University Hospital, Ostrava, Czech Republic
}

Address for correspondence: R. Zachoval, MD, PhD, Department of Urology, Thomayer Hospital and First Faculty of Medicine, Charles University, Videnska 800, CZ-140 59 Prague 4, Czech Republic.

Phone: +420.2.61083196

Acknowledgement: This project was supported by grant IGA of Ministry of Health of The Czech Republic NS/10431-3/2009. be deteriorated due to congenital or acquired abnormalities of the urogenital tract, urogenital infections, varicocoele, endocrine disorders, genetic abnormalities and immunological disorders (2). Deteriorated fertility in haemodialyzed patients with chronic kidney disease (CKD) is mainly caused by endocrine and immunological factors, the other factors may contribute to a lesser extent.

Fertility has a major impact on quality of life in men younger than 50 years of age. The above data show that there is a large group of patients with CKD on haemodialysis and impaired fertility in the Czech Republic. The aim of our study was to find out the incidence of hormonal and fertility disorders in patients less than 49 years of age with CKD on the waiting list for kidney transplantation and to define correlation between fertility disorders and the duration of haemodialysis.

\section{Methods}

All patients from 18 to 49 years of age with CKD, awaiting kidney transplantation and signed informed consent form for the study were examined from September 2009 to April 2011. These patients were recruited from haemodialysing centres (40 centres) which closely cooperate with Transplantcentre at IKEM Prague (Institute for Clinical and Experimental Medicine). Patients with diabetes mellitus and medications which can influence level of sex hormones or spermatogenesis were excluded from the study. 
$711-715$

List of performed tests and analysed data in patients group:

1) Causes of CKD - divided into 4 groups:

1.1 Glomerulonephritis

1.2 Tubulointerstitial nephritis

1.3 Polycystic kidney disease

1.4 Other

2) Time from the onset of CKD

3) Duration of haemodialysis

4) Serum concentrations of hormones (blood sampling was carried out according to the methodology of the European Association of Urology)

4.1 Testosterone (T) (standard 9.0 to $55.0 \mathrm{nmol} / \mathrm{l}$ )

4.2 Steroid hormone binding globulin (SHBG) (standard 11.2 to $78.1 \mathrm{nmol} / \mathrm{l})$

4.3 Luteinizing hormone (LH) (standard 1.2 to $8.8 \mathrm{IU} / \mathrm{l}$ )

4.4 Follicle stimulating hormone (FSH) (standard 0.95 to 9.7 IU/1)

4.5 Prolactin (PRL) (standard 72.7 to $407.4 \mathrm{mIU} / \mathrm{l}$ )

5) Semen analysis (sampling was performed according to WHO methodology):

5.1 Semen volume (standard $\geq 1.5 \mathrm{ml}$ )

5.2 Total sperm count (normal $\geq 39$ million)

5.3 Sperm concentration (normal $\geq 15 \mathrm{million} / \mathrm{ml}$ )

5.4 Total sperm motility (standard $\geq 40 \%$ sperm moving with grade $\mathrm{A}+\mathrm{B}+\mathrm{C}$ )

5.5 Progressive sperm motility (standard $\geq 32 \%$ sperm moving with grade $\mathrm{A}+\mathrm{B}$ )

5.6 Sperm morphology (standard $\geq 4 \%$ of normally shaped sperm)

The control group consisted of men from 18 to 49 years examined at urology department for stone disease from September 2009 to April 2011, and signed informed consent form to be included in the study. The same tests (hormone levels and semen analysis) were performed in the control group of patients.

Following parameters were evaluated:

1) Differences between the group of patients and controls:

1.1 hormone levels

1.2 semen analysis parameters

2) Correlations within the group of patients:

2.1 hormone levels

2.2 parameters of semen analysis

2.3 hormone levels and parameters of semen analysis

2.4 hormone levels and parameters of semen analysis and the cause of CKD

2.5 hormone levels and parameters of semen analysis and duration of $\mathrm{CKD}$

2.6 hormone levels and parameters of semen analysis and duration of haemodialysis

T-tests were used to find a statistically significant difference between the monitored normally distributed variables, between healthy controls and patients. Pearson's chi-squared test was used for categorical variables, i.e. to determine whether there was a statistically significant difference between the number of patients and healthy controls with normal and pathological values. The correlation between variables within the group of patients was evaluated using Pearson's correlation coefficient. The results were considered as statistically significant when $\mathrm{p}<0.05$.

\section{Results}

The group of patients consisted of 74 men, mean age 40 years $(\mathrm{SD} \pm 7.5)$ and control group of 41 men, mean age 36.7 years ( $\mathrm{SD} \pm 8.8)$. Glomerulonephritis was the cause of CKD in 20 patients $(28.2 \%)$, tubulointerstitial nephritis in 19 patients $(26.7$ $\%)$, polycystic kidney disease in 10 patients $(14.1 \%)$ and other aetiology in 22 patients (31\%). The average duration of CKD and haemodialysis in investigated patients was $130.4(\mathrm{SD} \pm 87.7)$ and $48.8(\mathrm{SD} \pm 41.7)$ months. Only 14 patients were treated with haemodialysis less than 1 year, 15 patients less than 2 years, 11 patients less than 3 years, 15 patients less than 4 years and 16 patients more than 4 years.

1) Comparison between the group of patients and control group:

1.1 hormone levels

Average testosterone levels were lower in patients compared to control group. Patients were found higher mean SHBG, LH, FSH and PRL levels than in the control group and the difference was statistically significant (Tab. 1).

Tab. 1. Differences between the group of patients and controls in hormone levels.

\begin{tabular}{lccccc}
\hline & $\mathrm{T}$ & SHBG & LH & FSH & PRL \\
\hline patients & 10.0 & 36.4 & 11.6 & 8.8 & 620.2 \\
controls & 13.9 & 30.0 & 3.1 & 3.2 & 290.8 \\
p & 0.090 & $\mathbf{0 . 0 3 0}$ & $\mathbf{0 . 0 0 3}$ & $\mathbf{0 . 0 0 6}$ & $\mathbf{0 . 0 0 1}$ \\
\hline
\end{tabular}

Bold: statistically significant

Kurz: values out of physiological range

Tab. 2a - d. Difference in number of patients and controls with normal and pathological hormonal levels.

2a) testosterone

\begin{tabular}{lcc}
\hline & normal T & decreased T \\
\hline patients (\%) & $50(70.4 \%)$ & $21(29.6 \%)$ \\
controls (\%) & $100(100.0 \%)$ & $0(0.0 \%)$ \\
\hline p & $\mathbf{0 . 0 0 6}$ & \\
\hline
\end{tabular}

2b) $\mathrm{LH}$

\begin{tabular}{lccc}
\hline & normal LH & decreased LH & increased LH \\
\hline patients (\%) & $42(59.2 \%)$ & $0(0.0 \%)$ & $29(40.8)$ \\
controls (\%) & $39(95.1 \%)$ & $2.0(4.9 \%)$ & $0(0.0 \%)$ \\
\hline $\mathrm{p}$ & $\mathbf{0 . 0 0 1}$ & & \\
\hline
\end{tabular}

2c) FSH

\begin{tabular}{lccc}
\hline & normal FSH & decreased FSH & increased FSH \\
\hline patients (\%) & $55(77.5 \%)$ & $0(0.0 \%)$ & $16(22.5 \%)$ \\
controls (\%) & $39(95.1 \%)$ & $2(4.9 \%)$ & $0(0.0 \%)$ \\
\hline p & $\mathbf{0 . 0 0 1}$ & & \\
\hline
\end{tabular}

2d) prolactin

\begin{tabular}{lccc}
\hline & normal PRL & decreased PRL & increased PRL \\
\hline patients (\%) & $37(52.1 \%)$ & $1(1.4 \%)$ & $33(46.5 \%)$ \\
controls (\%) & $33(80.5 \%)$ & $8(19.5 \%)$ & $0(0.0 \%)$ \\
\hline p & $\mathbf{0 . 0 0 1}$ & & \\
\hline
\end{tabular}

Bold: statistically significant 
Tab. 3. Differences between the group of patients and controls in semen analysis parameters.

\begin{tabular}{lcccccc}
\hline & semen volume & total sperm count & sperm concentration & total sperm motility & progressive sperm motility & sperm morphology \\
\hline patients & 2.1 & 52.9 & 19.9 & 39.3 & 16.2 \\
controls & 3.3 & 201.4 & 60.8 & 58.7 & 50.7 \\
\hline $\mathrm{p}$ & $\mathbf{0 . 0 1 1}$ & $\mathbf{0 . 0 0 1}$ & $\mathbf{0 . 0 0 1}$ & $\mathbf{0 . 0 0 1}$ & $\mathbf{0 . 0 0 1}$ \\
\hline
\end{tabular}

Bold: statistically significant

Kurz: values out of physiological range

Tab. 4. Correlations within the group of patients: hormone levels and parameters of semen analysis.

\begin{tabular}{lccccc}
\hline & T & SHBG & LH & FSH & PRL \\
\hline semen volume & 0.990 & 0.859 & 0.100 & $\mathbf{0 . 0 0 1}$ & 0.864 \\
total sperm count & 0.398 & 0.638 & 0.638 & 0.122 & 0.440 \\
sperm concentration & 0.146 & 0.254 & 0.674 & 0.771 & 0.184 \\
total sperm motility & $\mathbf{0 . 0 1 9}$ & 0.732 & 0.574 & 0.583 & 0.896 \\
progressive sperm motility & 0.077 & 0.274 & 0.313 & 0.409 & 0.636 \\
sperm morphology & $\mathbf{0 . 0 2 4}$ & 0.467 & $\mathbf{0 . 0 4 8}$ & 0.311 & 0.753 \\
\hline
\end{tabular}

Bold: statistically significant

Significant differences for all hormones except SHBG were found, when the numbers of patients and healthy individuals with normal and pathological values of hormones were compared (Tab. 2a-d).

1.2 semen analysis parameters

Decreased mean volume of ejaculate, total sperm count, sperm concentration, total and progressive sperm motility and sperm mor-

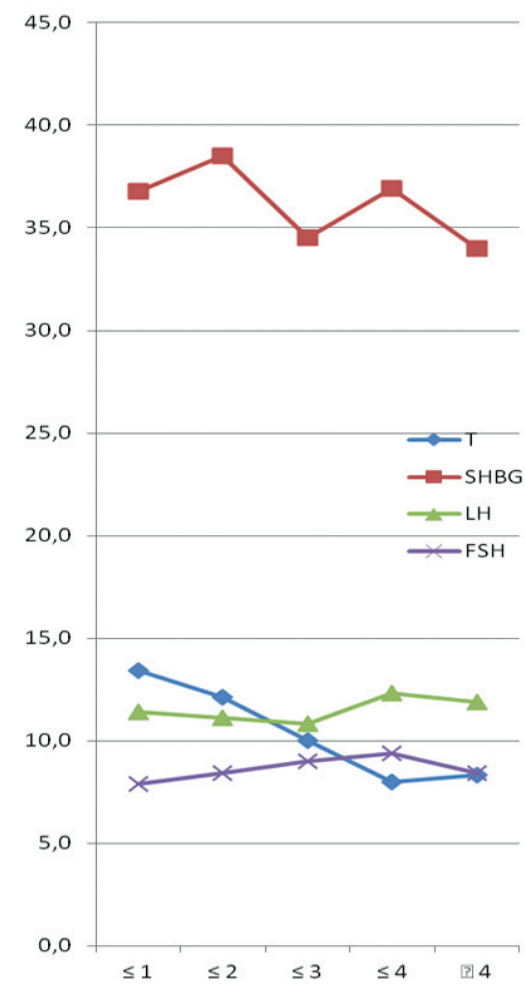

Fig. 1. Dynamics of hormone levels in dependence on the duration of haemodialysis. Axis x: years, Axis y: concentration of hormones. phology were found in patients compared to the control group and the difference was statistically significant (Tab. 3).

2) Correlations within the group of patients:

2.1 hormone levels

Negative correlation was identified between T and PRL plus between SHBG and T + FSH. Positive correlation was indentified between $\mathrm{LH}$ and FSH.

\subsection{Parameters of semen analysis}

Positive correlation was identified between almost all parameters of semen analysis except ejaculate volume, which correlated only with total sperm count.

2.3 hormone levels and semen analysis parameters

Positive correlation was identified between $\mathrm{T}$ and total sperm motility and morphology, between LH and sperm morphology, and further between FSH and volume of ejaculate (Tab. 4).

Correlation was not identified between hormone levels, semen analysis parameters and the cause of CKD and further no correlation was found between hormone levels, semen analysis parameters and the duration of CKD.

2.4 hormone levels, semen analysis parameters and duration of haemodialysis

Negative correlation was identified between the duration of haemodialysis and T (Fig. 1).

Negative correlation was identified between the duration of haemodialysis and sperm concentration, total and progressive motility and sperm morphology (Fig. 2).

\section{Discussion}

According to World Health Organization about $15 \%$ of couples are not able to conceive within one year and seek for infertility

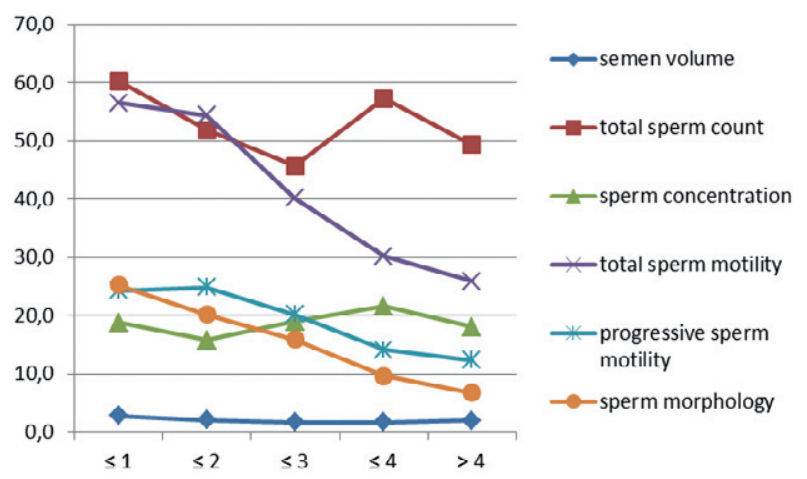

Fig. 2. Dynamics of semen analysis parameters in dependence on the duration of haemodialysis. Axis x: years, Axis y: parameters of spermiogram. 
treatment. Approximately $5 \%$ of couples remain without their own offspring despite treatment of infertility. The male infertility factor (abnormalities in semen analysis: oligozoospermia, asthenozoospermia and teratozoospermia) causes infertility in $50 \%$ of infertile couples. Male fertility may be reduced due to congenital or acquired abnormalities of the urogenital tract, urogenital infections, varicocoele, endocrine disorders, genetic abnormalities and immunological disorders. Idiopathic male infertility involves $30-40 \%$ of infertile men and the cause of infertility cannot be identified in these cases (2).

Data about the incidence of CKD are inaccurate and practically undetectable. There are high numbers of patients with CKD who are not diagnosed at all, usually due to minor clinical symptoms. Estimated incidence of CKD is about $5 \%$ of the population in the USA (3) for intermediate (grade 3) plus severe (grade 4) i.e. calculated glomerular filtration with $<0.5 \mathrm{~mL} / \mathrm{s}$ ). The incidence of end stage (grade 5) CKD, when the treatment of renal failure with dialysis or renal transplantation is necessary, is easily traceable due to records of patients in dialysis and transplant centers. Dialysis care was provided for 6318 patients in the Czech Republic at the end of 2010 i.e. 602 per 1 million inhabitants. Haemodialysis was performed in 5820 patients and peritoneal dialysis in 498 patients. Number of patients dependent on haemodialysis increases every year, in 2001 it was 379 patients per 1 million inhabitants and in 2010 already 554 patients per 1 million inhabitants. Of the total number of haemodialyzed patients $30 \%$ (1746 patients in 2010 ) is under the age of 60 years and approximately $20 \%$ (1164 patients in 2010) dialyzed patients should be considered for renal transplantation according to current medical standards (1).

Fertility deterioration in haemodialyzed patients with CKD is mainly due to endocrine and immunological factors, to a lesser extent other factors may be involved as well. Fertility has a great importance mainly for men less than 50 years of age. The above data show, that there is a large group of haemodialyzed patients with CKD and impaired fertility in the Czech Republic and elsewhere in the world as well. The subgroup of patients with the most favorable prognosis is represented by the patients on a waiting list for kidney transplantation. These patients are on average younger with less comorbidities and they have the greatest interest for fertility preservation or treatment of infertility.

Chronic kidney disease leads to infertility in men, which may manifest by the reduction of all basic parameters of semen analysis: ejaculate volume, sperm count and concentration, reduced motility and impaired sperm morphology. Both qualitative and quantitative parameters of semen analysis depend on the endocrine, metabolic and immunological environment of the organism, and the hormone levels are the most important factors affecting spermatogenesis in patients with CKD requiring dialysis (1).

It has been repeatedly demonstrated that $\mathrm{T}$ level decreases in haemodialysis patients, it is highly probable due to dysfunction of Leydig cells induced by CKD and haemodialysis (4). As a result of low testosterone level LH increases, however deteriorated clearance may participate on this process as well (5). The fact, that low therapeutic dose of $\mathrm{T}$ causes a significant decrease or normalization of LH level in haemodialyzed patients with im- paired fertility, may be considered as a prove at least temporary preserved function of hypothalamic-pituitary-testicular axis in these patients (6). Elevated PRL level is another factor that may contribute to a decrease of testosterone. Hyperprolactinemia occurs in patients with CKD very often and mainly is caused by reduced clearance of PRL. Further, some other well-known factors may also contribute to hyperprolactinemia (7). A causal link between hyperprolactinemia and low testosterone level is documented in study with dopamine agonist bromocriptine, which is used for treatment of hyperprolactinemia. Bromocriptine administration in patients with CKD leads to an increase of pathologically low $\mathrm{T}$ levels, without significant impact on elevated levels of LH-RH and LH. The effect of hyperprolactinemia on Leydig cells is direct and not mediated by affecting the hypothalamic-pituitary-testicular axis (8).

Decreased quality of semen analysis and low testosterone level is usually associated with increased level of FSH (5). The fact, that therapeutic dose of $\mathrm{T}$ leads to an improvement of semen analysis parameters and normalization or significant decrease of FSH level in some patients, may be considered as a prove at least temporary preserved function of hypothalamic-pituitary-testicular axis in dialyzed patients with impaired fertility (6).

Kidney transplantation is the most effective treatment of the end stage CKD. Renal transplant patients have a significantly longer survival and a significantly lower morbidity compared to dialyzed patients (9). Chronic kidney disease leads to a development of a number of pathophysiological mechanisms in a human body relating to virtually every organ and functional systems, such as cardiovascular, metabolic, endocrine, etc. Renal transplantation has a potential to positively affect some of these patophysiological mechanisms, and some of the dysfunctions or their symptoms or consequences even restore (10). In this context, the impact of renal transplantation on fertility has been assessed in the past, unfortunately the results of these studies are not conclusive. While some authors have demonstrated the benefits of kidney transplantation for improvement of male fertility, including a tendency to normalize hormonal levels (11-13), the other authors have not proved the same result regarding male fertility $(14,15)$. In our work we monitored all basic fertility parameters in the group of patients with CKD requiring haemodialysis. In addition, we explored the issue, which has not been studied yet, and it is causal connection between the development of infertility and the time on the waiting list for renal transplantation. As expected, we found that the group of patients had statistically significantly lower all monitored semen analysis parameters and statistically significantly higher levels of LH, FSH and PRL compared with control group. The mean testosterone level was lower in the group of dialysed patients, but the difference was not statistically significant. This fact can be explained by finding $\mathrm{T}$ levels at the upper limit of normal range in some patients, who were also diagnosed to have very high levels of LH. Consequently we could assume, that there is a certain group of patients who are temporarily able to compensate low testosterone levels with high production of LH. Further, a statistically significant correlation was detected between hyperprolactinemia and low testosterone level in the 
group of patients and statistically significant correlation between low testosterone level and reduced sperm motility and abnormal sperm morphology, which represent the most sensitive qualitative parameters of the sperms.

An important result of the study is the finding that the duration of haemodialysis significantly correlated with low testosterone level and deterioration of most semen analysis parameters. A significant worsening of fertility parameters occurs after about 3 years of haemodialysis. This suggest, that early kidney transplantation less than three years from the start of haemodialysis, may prevent serious and often irreversible deterioration of fertility due to early restoration of hormonal levels and other functions of the organism.

\section{References}

1. Yearbook of dialysis in the Czech Republic in 2010. http://www.nefrol. cz/resources/upload/data/274_Rocenka2010.pdf

2. World Health Organization. WHO Manual for the Standardized Investigation and Diagnosis of the Infertile Couple. Cambridge University Press, 2000.

3. Viklický O. Chronické selhání ledvin a uremický syndrom. In: Tesař V, Schück O et al (Eds). Klinická nefrologie. Prague: Grada Publishing; 2006.

4. Bellinghieri G, Santoro D, Mallamace A, Savica V. Sexual dysfunction in chronic renal failure. J Nephrol 2008; 21 (Suppl 13): S113-117.

5. Vecchio M, Navaneethan SD, Johnson DW et al. Treatment options for sexual dysfunction in patients with chronic kidney disease: a systematic review of randomized controlled trials. Clin J Am Soc Nephrol 2010; 5 (6): 985-995.
6. van Coevorden A, Stolear JC, Dhaene M, van Herweghem JL, Mockel $\mathbf{J}$. Effect of chronic oral testosterone undecanoate administration on the pituitary-testicular axes of hemodialyzed male patients. Clin Nephrol 1986; 26 (1): 48-54.

7. Hou SH, Grossman S, Molitch ME. Hyperprolactinemia in patients with renal insufficiency and chronic renal failure requiring hemodialysis or chronic ambulatory peritoneal dialysis. Am J Kidney Dis 1985; 6 (4): 245-249.

8. Bry-Gauillard H, Touraine P, Mamzer-Bruneel MF et al. Complete regression of a major hyperprolactinaemia after renal transplantation. Nephrol Dial Transplant 1999; 14 (2): 466-468.

9. Tonelli M, Wiebe N, Knoll G et al. Systematic review: kidney transplantation compared with dialysis in clinically relevant outcomes. Am J Transplant 2011; 11 (10): 2093-2109.

10. Favi E, Salerno MP, Romagnoli J, Castagneto M, Citterio F. Significant improvement in patient survival after renal transplantation in the last decade. Transplant Proc 2011; 43 (1): 285-287.

11. Xu LG, Yang YR, Wang HW et al. Characteristics of male fertility after renal transplantation. Andrologia 2011; 43(3): 203-207.

12. de Celis R, Pedrón-Nuevo N. Male fertility of kidney transplant patiens with one to ten years of evolution using a conventional immunosuppressive regimen. Arch Androl 1999; 42 (1): 9-20.

13. Akbari F, Alavi M, Esteghamati A et al. Effect of renal transplantation on sperm quality and sex hormone levels. BJU Int 2003; 92 (3): 281-283.

14. Zuber J, Anglicheau D, Elie C et al. Sirolimus may reduce fertility in male renal transplant recipients. Am J Transplant 2008; 8 (7): 1471-1479.

15. Baumgarten SR, Lindsay GK, Wise GJ. Fertility problems in the renal transplant patient. J Urol 1977; 118 (6): 991-993.

Received March 22, 2012. Accepted September 20, 2013. 